\title{
Personalidad: un recorrido por los principales conceptos desarrollados
} sobre el constructo

Personality: a tour of the main concepts developed about the

Para referenciar este artículo:

Grasso, P. (2018). Personalidad: un recorrido por los principales conceptos desarrollados sobre el constructo. Conciencia EPG, 3(2), 34-57.doi: $10.32654 /$ CONCIENCIA EPG.3-2.3

\section{Resumen}

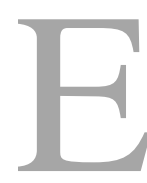

1 presente trabajo tiene como objetivo presentar un recorrido articulado acerca de los trabajos más importantes sobre la personalidad. Si bien se trata de una variable muy conocida, es -al mismo tiempo- una variable que refiere una estructura polisémica y, sobre la cual, aún no existe una idea unificada acerca de cómo debe ser tratada. Si bien esto último puede entenderse como parte intrínseca en cuanto a la naturaleza de la misma, es conveniente realizar desarrollos que traten de llegar a una idea en que se alcance una operacionalización unificada. En base a esto, se espera que el presente trabajo pueda dar cuenta de la cohesión de este constructo a partir de integrar distintas perspectivas.

Palabras clave: personalidad, rasgos, factores. 
Abstract

he present work aims to present an articulated journey about the most important developments about personality. Although it is a variable that is well known, it is - at the same time - a variable that refers to a polysemic structure, and about which there is not yet a unified idea about how it should be unified. Although the latter can be understood as an intrinsic part in terms of the nature of the same, it is convenient to carry out developments that try to arrive at an idea that encompasses a unified operationalization. Based on this explained, it is expected that this work can account for the cohesion of this construct from integrating different perspectives.

Keywords: personality, traits, factors.

\section{Introducción}

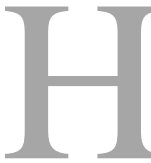
ace tiempo que la Psicología de la Personalidad trata de establecer los mecanismos fundamentales en que las personas difieren; así, se han desarrollado a través de los años numerosas taxonomías que refieren a los rasgos de personalidad y que permiten determinar las diferencias individuales en cuanto a dichos rasgos (Wilt \& Revelle, 2015). Para Eysenck (1947), la personalidad es la suma total -ya sea real o potencial- de los patrones de conducta de un organismo, entendiendo que la misma puede estar determinada por el ambiente o la herencia. Este autor sostiene que la personalidad se origina y desarrolla a través de la interacción de cuatro componentes principales: el sector conativo, el cognitivo, el somático y el afectivo.

Con el fin de explicar este concepto, se han desarrollado diferentes modelos teóricos, a partir de los cuales pueden ser comprendidos muchos de los actos y conductas manifestadas por los seres humanos, y que permiten a su vez explicar los rasgos de personalidad. En cuanto a los factores se entienden como independientes entre sí y brindan información de la variación fenotípica de la personalidad. En términos generales, estos factores son: extraversión, agradabilidad, responsabilidad, neuroticismo y apertura a la experiencia (Castro Solano \& Casullo, 2001; Mc Crae \& Costa, 1987; Schmitt, Allik, Mc Crae \& Bennet Martínez, 2007).

\section{Personalidad: recorrido histórico y una aproximación al concepto}

La personalidad se encuentra entre los constructos más estudiados y discutidos en del campo de la Psicología; muchos son los autores (Allport, 1937, 1970a, 1970b; Cattell, 1979; Eysenck, 1947, 1991; McCrae \& Costa, 1987; Mischell, 1973, 1988) que trataron, desde diferentes posturas teóricas, dar una definición que considere todos los aspectos posibles. No obstante, aún no existe una definición unificada de este concepto, en 
cambio la revisión de la literatura científica muestra una gran variedad de definiciones (Chico Librán, 2015).

Desde la antigua Grecia se estudiaba la personalidad, y es en esa época donde surge el término derivado etimológicamente del concepto de persona (del griego prosopon), que hacía referencia a cara, rostro, máscara. Teniendo en cuenta esto último, las personas eran las máscaras usadas por los actores en el teatro cuando debían representar los distintos personajes, es decir, las usaban para caracterizarlos, dando una imagen externa específica, que era también superficial, sólo tenía como fin contextualizar; sin embargo, aunque en un principio estuviese más relacionado a la apariencia, al paso del tiempo ha ido cobrando un significado más profundo, asociándose con los aspectos más estables y determinantes de los individuos (Mikulic, s.f.; Montaño Sinisterra, Palacios Cruz \& Gantiva Díaz, 2009).

En resumen, puede decirse que lo que refiere a la personalidad estaba ligado a las características más visibles y externas, es decir, a aquellos aspectos que pueden ser observados a simple vista por los otros; sin embargo, al momento de hablar de la personalidad, tal como es conocida en la actualidad, se entiende que se trata de conjunto de características (no solo externas sino también internas) que dan cuenta de la forma de ser de un sujeto, a la vez, que permite diferenciarlo de otro (Chico Librán, 2015).

Por ejemplo, Cicerón (106-43, citado en Montaño Sinisterra, Palacios Cruz \& Gantiva Díaz, 2009) hace una definición que integra cuatro aspectos: 1) la forma en la cual los sujetos se presentan ante el resto; 2) el rol que desempeña en su vida; 3) las cualidades que lo conforman; y 4) según el nivel social al cual se pertenecía era sinónimo de dignidad y prestigio. De esta forma, Montaño Sinisterra et al. (2009) sostienen que, se sentaron las bases para las definiciones que siguieron en el tiempo sobre este constructo, ya que estas características pueden ser observadas en las personas $\mathrm{y}$, en general, esos atributos observados van siempre desde lo más externo a lo más interno.

Otra de las perspectivas asociadas a la era clásica es la de la Santísima Trinidad, que entiende a la personalidad desde un lugar sustancial y no asumido, lo que significa que viene como algo dado, que es esencial al ser humano; no obstante, más entrando la era medieval, se fueron agregando al concepto (casi de forma más novedosa), por un lado, aspectos éticos y, por otra parte, cualidades más distintivas del individuo, a partir de lo cual permitió perfilar de mejor forma al sujeto como tal, y no de manera tan general (Cerdá, 1985; Montaño et al., 2009).

A partir de esto, los términos persona y esencia fueron relacionados por los 
primeros teólogos, dando como resultado la primera definición integrada de personalidad, desde la que se entiende "a la persona como una sustancia individual, racional y natural" (Montaño et al., 2009, p. 83). Esto permitió a su vez, que, por un lado, se incluyese la racionalidad como atributo, $\mathrm{y}$, por otro, apareciesen diferentes definiciones filosóficas, a las que más tarde se sumaran las psicológicas (Allport, 1970 a, b). Los planteos desde esta última disciplina se refieren a distintas cualidades que son parte de cada individuo (Leal, Vidales \& Vidales, 1997).

Por todo lo mencionado, se puede esbozar una primera aproximación, aunque general, a la definición de la personalidad, entendiéndola como una organización dinámica de las fuerzas, tanto psicológicas como físicas, que permiten que el individuo se adapte al medio ambiente (Allport, 1937). Complementando esta definición se puede tomarse la idea de Brenlla (1996), quien hace referencia a esas características que se mantienen en el tiempo y que determinan la conducta de los individuos.

En función de estas ideas, se puede arribar a una definición más amplia en cuanto a lo que este concepto hace referencia en el área de la Psicología. Así, se retoma la definición de Bermúdez (1999), ya que la misma integra los aspectos antes mencionados, y quien sostiene que se trata de una organización que es relativamente estable, integrada de características estructurales y funcionales, así como adquiridas e innatas, que se desarrollan según las condiciones específicas de cada contexto y forman un conjunto único de conductas con las que cada sujeto afronta cada situación.

Ahora bien, es necesario aclarar que, si se ha llegado a definiciones más complejas, es porque el estudio -en generalde este concepto se ha ido complejizando. En ese sentido, se ha observado que, más allá de las diferentes definiciones y/o teorías en las cuales se enmarquen dichas definiciones, todas tienen los siguientes puntos en común:

i) La personalidad está compuesta de las conductas más observables (externas) así como de las más profundas (internas), entre las cuales están las emociones, y que no se pueden ver tan fácilmente;

ii) Las características desarrolladas en general son estables y también consistentes, lo que resulta importante ya que es lo que permite predecir la conducta; y

iii) Hace referencia a un carácter único, lo que implica una estructura configurada de forma también única, de un conjunto de elementos particulares en cada sujeto (Chico Librán, 2015).

Al mismo tiempo, Leal et al. (1997) y Chico Librán (2015) hacen mención de una categorización con respecto a las formas de 
definir la personalidad, que puede resumirse de la forma siguiente:

a) Definiciones aditivas: Son aquellas que suelen empezar con la frase "la personalidad es la suma de...'; algunas veces se reemplaza la palabra suma por conjunto, cúmulo. Lo importante es que, en cualquiera de los casos, la tendencia es a juntar los elementos. Respecto a esto, Allport (1966) las entiende como poco consistentes, ya que son solo una enumeración de elementos; Chico Librán (2015) aclara que, esto puede generar cierta confusión en cuanto si en verdad existe la personalidad como un fenómeno en sí mismo o si se usa este concepto para describir solo una serie de fenómenos.

b) Definiciones integrativas: En este caso, son un poco más complejas, ya que entiende a la personalidad como la integración de diferentes elementos que definen a un sujeto, y resaltan la importancia de la organización y estructuración de esos elementos; al mismo tiempo, esa organización es única, ya que depende de la interrelación con el ambiente, lo que da como resultado un sujeto de características únicas. En algunos casos, se consideran aquellas que aceptan que la personalidad puede cambiar según la etapa de desarrollo del sujeto. c) Definiciones jerárquicas: Estas definiciones también tienen un carácter integrativo, solo que, a diferencia de las anteriores, entienden que hay ciertos elementos que predominan sobre otros; ponen énfasis en el carácter estructural y de ordenamiento que ocupa cada elemento, tomando estos últimos un significado específico en la integración de la personalidad.

d) Definiciones en términos de ajuste: Hacen referencia a la capacidad adaptativa de la personalidad; es decir, refieren al conjunto integrado y ya organizado de elementos en los individuos, y a cómo este conjunto va a ajustarse al contexto.

e) Definiciones basadas en la distintividad: Resaltan como personalidad aquello que es más distintivo, esencial y peculiar de cada individuo; en ese sentido la personalidad se asocia a aquello que hace a los individuos diferenciarse unos de otros.

\section{Temperamento y carácter}

Cabe aclarar que hay dos conceptos que se asocian a la personalidad, pero que, si bien le dan sustento, no son sinónimos; incluso en la jerga más coloquial existe una tendencia a tratarlos como iguales, pero en el ámbito científico no son lo mismo. Se trata del carácter y el temperamento. 
En cuanto al carácter, deriva del latín character y del griego charaktér y significa hacer una incisión, marcar; a partir de esto, entre las tantas definiciones que evidencia la Real Academia Española (2017) se destacan las siguientes:

- Señal o marca que se imprime, pinta o esculpe en algo;

- Conjunto de cualidades o circunstancias propias de una cosa, de una persona o de una colectividad, que las distingue, por su modo de ser u obrar, de las demás; e, incluso:

- Marca o hierro con que los animales de un rebaño se distinguen de los de otro.

Como puede observarse, de todas estas se entiende la idea de que se trata de alguna marca o especificidad que hace que las cosas, o los sujetos, puedan ser diferenciados unos de otros.

Para la Psicología, su significado serían aquellas características que se forman de procesos históricos y sociales que atraviesan a un sujeto, a partir del cual se configura la especificidad que, a su vez, genera el modo de ser individual. En ese sentido, involucra a todas las características que se adquieren a lo largo del crecimiento, y, al mismo tiempo, supondría un determinado grado de conformidad con las reglas sociales establecidas en el contexto de desarrollo (Chico Librán, 2015; Montaño et al., 2009; Orengo Caus et al., 2007).
Así, el carácter se desarrolla a lo largo de la vida, a partir de un conjunto de situaciones neuropsíquicas (es decir las funciones psíquicas en relación con el sistema nervioso), en función de las actividades que aparecen en el devenir mismo de la vida, así como las actitudes sobre estas actividades, que son parte de la constante adaptación a las condiciones socio-ambientales (Chico Librán, 2015). Lluís Font (2002) y Montaño et al. (2009) sostienen que el carácter no sólo controla, sino que también modifica y es autoregulador de la actividad de los sujetos para que estos puedan brindar respuestas adecuadas a las exigencias del contexto.

Algo más para decir sobre este término, es que, los elementos que lo componen se establecen en una unidad que es coherente, manteniendo cierto grado de uniformidad a la hora de expresar las manifestaciones sobre los cambios que suceden alrededor del individuo (Chico Librán, 2015).

En resumen, se ha esbozado que el sello personal o el estilo de vida que se manifiesta de forma específica o individual en las personas es la contribución social a la formación de la personalidad.

Sobre el temperamento, siguiendo a Millon y Everly (1994) éste sería la conformación biológica de la personalidad (tanto en lo que refiere a los aspectos emocionales como a los motivacionales) y, por tanto, viene predeterminada. Castro 
Solano (2015) aclara que, incluye los aspectos menos influidos por el ambiente, que también son los "de naturaleza más hereditaria" (p. 23). No solo involucra lo hereditario y congénito, sino también, estímulos más externos como ambiente, alimentación, clima, entre otros. No obstante, sigue siendo la parte de la personalidad más refractaria en lo que al cambio refiere; a pesar de ello puede ser modificable dentro de determinados límites, ya que intervienen factores contextuales; es decir, que los elementos genéticos que lo componen se modelan según en contexto (Chico Librán, 2015; Orengo Caus et al., 2007). Montaño et al. (2009) sintetizan de la siguiente manera: "el temperamento es un fenómeno naturalmente emocional, es decir, que se puede presentar a causa de factores genéticos o hereditarios, pues los individuos reaccionan de manera rápida e intensa a la estimulación ambiental” (p. 85).

Por otro lado, es también un término que cuenta con cierta tradición histórica. Al respecto, Chico Librán (2015) se apoya en Hipócrates para explicar la distinción entre cuatro tipos de temperamentos: el colérico, el sanguíneo, el melancólico y el flemático, explicando que estas formas de reaccionar estaban asociadas a fuentes somáticas.

En cuanto a los dos primeros tienen que ver con la forma fácil de excitabilidad, así como con una rápida fluctuación de los intereses (en el colérico son débiles y en el sanguíneo son intensos); en los dos restantes la excitabilidad es lenta y persistente (en el caso del flemático es débil, mientras que en el melancólico es fuerte). Lo que resulta interesante es que, esta clasificación ha llegado hasta estos días, sirviendo de base a las diferentes tipologías que se han ido desarrollando, aun cuando estas últimas difieran en función de los modelos propuestos.

En definitiva, el temperamento podría entenderse como la estructura sobre la que se desarrolla la personalidad. Castro Solano (2015) explica que "los autores contemporáneos determinaron que alrededor de un $40 \%$ de las diferencias individuales de la personalidad se deben a diferencias temperamentales heredadas" (p. 23).

De esta forma, ha quedado expuesto que los términos carácter y temperamento no son sinónimos de la personalidad, aunque sí su interacción es la misma (Figura I). Para Lluís Font (2002), las influencias psicosociales y culturales influyen al momento de configurar la personalidad específica de cada individuo, aunque estos factores interactúan con los sistemas más profundos de la naturaleza humana.

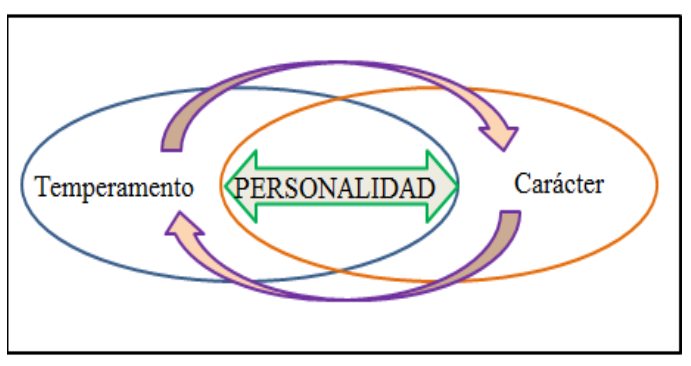

Figura 1. Interacción entre temperamento y carácter como componentes de la personalidad 
Tipo y rasgo: dos formas de describir la personalidad

De la misma forma que ha ido adquiriendo la complejidad este constructo, se hace necesaria su distinción con respecto a términos, como tipo y rasgo.

Cuando se habla de rasgos se hace referencia a las características específicas de una persona sobre las cuales se distingue de otra, al mismo tiempo, esto provoca que los comportamientos también sean distintos en unos y en otros; se puede decir que alguien posee algún grado de determinado rasgo, desde mucho hasta poco (Cloninger, 2003). Según Castro Solano (2015) serían las tendencias latentes que hacen que los sujetos se comporten de una manera determinada; los rasgos también son los responsables en cuanto a las diferencias individuales dado que se puede predecir la conducta en situaciones distintas. Para este autor no son observables sino de naturaleza inferencial.

Con respecto a sus características, se pueden distinguir dos principios fundamentales: por un lado, la estabilidad y por otro la consistencia, a partir de lo que se entiende que hay algo que es duradero en el tiempo, que se observa más allá de las distintas situaciones (Castro Solano, 2015). Cloninger (2003) sostiene que, a partir de los rasgos puede realizarse una descripción bastante precisa de la personalidad, ya que las características agrupadas son más específicas; el hecho de la gradación también colabora en esa especificidad.
En lo que refiere a los tipos, Cloninger (2003) los define como a las categorías de personas que cuentan con características que se parecen. En ese sentido, bastan solo unos pocos tipos para describir a la gente; dicho de otra manera, cada individuo es parte de un tipo o no lo es. La autora también agrega que el hecho de pertenecer a una categoría específica dice mucho de cada miembro, y al mismo tiempo existe la idea de que las personas entren de manera automática en categorías naturales. Castro Solano (2015) retoma la idea de rasgo y dice que "la agrupación de distintos rasgos se denomina tipo" (p. 19); la diferencia, entonces, con el rasgo es que el tipo es categorial y alude a un perfil.

En resumen, se ha dicho que la personalidad generalmente es descrita en función de estos dos conceptos: tipo y rasgo; en cuanto al primero, usualmente es utilizado para describir a un individuo en función de una característica específica, mientras que el segundo supone una graduación promedio entre características opuestas; para citar un ejemplo, en el caso de los tipos una persona sería introvertida o extrovertida, en el caso del rasgo habría una tendencia hacia alguna de estas características pero ambas estarían presentes en el individuo (Eysenck, 1947, 1991).

Por todo lo señalado, se puede entender la multiplicidad de definiciones acerca de este constructo, así como el hecho de que se asocien de forma tan estrecha tanto 
en el desarrollo evolutivo de los individuos como con sus conductas, llegando en algunos casos a relacionar a la personalidad con un conjunto de rasgos, en otros a un repertorio de conductas, e incluso -en otros casos- a una estructura organizada e integrada tanto a la acción como a la experiencia (Costa \& McCrae, 1995). Sumado a lo anterior, se desprende que este modo de ser único no solo permite diferenciar a los individuos entre sí, sino que también permite conocer sus motivaciones al momento de pensar, sentir, actuar y desenvolverse en su medio de una forma determinada (Seelbach González, 2012). En esta misma línea, Castro Solano (2015) señala que hay autores contemporáneos que también incluyen distintos aspectos, entre los que pueden observarse los estilos cognitivos, afrontamiento, representaciones sociales, procesos básicos, entre otros.

En consecuencia, puede decirse que el estudio de la personalidad tiene como objetivo principal el análisis de las diferencias individuales; al mismo tiempo, hace referencia a la persona de forma general a la vez que identifica las características más profundas y estables que conforman el núcleo de la identidad individual.

\section{Teorías de la personalidad}

En este apartado, se describen diferentes modelos que teorizan acerca de la personalidad. Para eso, primero se retoma la explicación que da Castro Solano (2015), quien -a su vez- sigue la sistematización realizada por Millon (1996), ya que antes de explicar las diferentes teorías parece apropiado entender los diferentes tipos de taxonomías a los que éstas adhieren. De esta manera, se describen los enfoques monotaxonómicos y politaxonómicos, que a su vez cada uno se divide en explicativo y empírico; luego se desarrollan los conceptos fundamentales de las teorías: psicodinámicas, humanísticos-existenciales, cognitivas-aprendizaje social y biológicasde rasgos.

\section{Enfoques monotaxonómicos y enfoques politaxonómicos}

Siguiendo la explicación de Castro Solano (2015), que realiza a partir de la propuesta de Millon, la sistematización taxonómica involucra dos enfoques: el monotaxonómico y el politaxonómico; estos también se dividen en dos formas cada uno: una explicativa y otra empírica (Figura II). En cuanto al enfoque monotaxonómico son aquellos que solo se ocupan de pocas unidades de análisis, y no tienen como finalidad explicar todo el desarrollo de la personalidad, sólo tienen en cuenta aquellas áreas que predeterminaron estudiar. Los enfoques politaxonómicos, a diferencia del anterior, buscan explicaciones que permitan dar cuenta de la estructura de la personalidad, para entender las diversas variaciones que pueden observarse en la conducta de los individuos a partir de un número pequeño de variables, o de algún principio teórico rector. 


\begin{tabular}{|c|c|}
\hline $\begin{array}{c}\text { MONOTAXONÓMICO } \\
\text { EMPIRICO }\end{array}$ & $\begin{array}{c}\text { MONOTAXONÓMICO } \\
\text { TEÓRICO }\end{array}$ \\
\hline POLITAXONÓMICO & POLITAXONÓMICO \\
EMPIRICO & TEÓRICO \\
\hline
\end{tabular}

Figura 2. Diferentes enfoques teóricos en los que pueden clasificarse las diferentes teorías de la personalidad

\section{Enfoque monotaxonómico - empírico}

Se trata de pocas categorías de análisis o solo una. Parte de la idea de que el conocimiento se obtiene de los datos, que se obtienen de los instrumentos de medición, que ya fueron desarrollados y no analizando nuevos conceptos. En general, lo que se hace es descomponer los instrumentos utilizados en sus partes, y los elementos extraídos son los que representan los distintos aspectos de la realidad, tal y como son en esta misma; esto último implica que exista una alta correspondencia entre las variables observadas y las latentes. Una limitación en este enfoque viene dada por tener que elegir cuáles variables explican de mejor forma y/o son necesarias para describir la personalidad, ya que las variaciones en esta misma se explican en función de pocos rasgos; a partir de esto último se puede resaltar que son parte de este enfoque las teorías tradicionales del rasgo. Los desarrollos de Cattell y Eysenck pueden ser ubicados en esta perspectiva (Castro Solano, 2015).

\section{Enfoque monotaxonómico - teórico}

Estos enfoques parten de supuestos teóricos para tratar conceptos que son no observables (o inferenciales). También se trabaja con pocas unidades de análisis que puedan explicar el objeto de estudio elegido. En este tipo pueden observarse los desarrollos de corte psicoanalítico, como por ejemplo los de Kernberg y Kohut.

\section{Enfoque politaxonómico - empírico}

Este enfoque, de manera parecida al monotaxonómico empírico, también parte del uso de instrumentos de medición, los cuales deben dar cuenta del isomorfismo entre estos últimos y la realidad. No se consideran los avances teóricos, sino que parte de un ajuste psicométrico de lo instrumental ya existente; en este caso no se basa en la descomposición de unidades de análisis para poder obtener, así, una mayor especificidad sobre de estas mismas. Se apoya en la idea de que las grandes diferencias entre los individuos están codificadas en el lenguaje natural. Este enfoque no toma, a diferencia del anterior, los elementos no observables. En este caso sería la teoría del Big Five (Costa y McCrae, 1985) la que mejor representa este enfoque.

\section{Enfoque politaxonómico - teórico}

Se trata de aquellos modelos integradores que tienen como objetivo desarrollar una estructura teórica taxonómica que dé cuenta de una clasificación de los distintos tipos de personalidad, así como de sus desórdenes; en ese sentido, se espera que una teoría pueda dar una explicación integrada y acabada del fenómeno que se estudia. A partir de esto se 
espera que pueda proveer de un sistema de diagnóstico en función de la realidad. Otra de las características a esperar es la existencia de relación entre las unidades de análisis que la componen, porque permite conceptualizar los términos que se usan en su estudio. El desarrollo mejor identificado en este enfoque es la teoría de Theodore Millon, la que -incluso- puede considerarse un puente entre la personalidad normal y la patológica.

Estos enfoq|ues permiten observar, de una manera general, la forma en la que suelen agruparse las teorías de la personalidad, lo que brinda una perspectiva de cómo deben entenderse. A continuación, se describen algunas de las teorías más representativas acerca de la personalidad.

\section{Teorías de la personalidad más representativas}

\section{A. Teorías Psicodinámicas}

En el grupo de teorías que son incluidas en este enfoque se observa que la perspectiva central es el estudio del inconsciente; a partir de esto se desprende que las teorías que conforman este grupo son las psicoanalíticas, ya sean las más tradicionales o las más modernas.

Morris y Maisto (2005) explican que la conducta de los individuos resulta de diversas fuerzas psicológicas internas, que operan en lo profundo de los sujetos, y que se dan afuera de la conciencia. Entender este mecanismo es vital ya que a partir de esto se puede comprender la personalidad. Cloninger (2003) pone de manifiesto que los individuos no estarían conscientes de los determinantes que más influyen en su forma de comportarse. En ese sentido puede esperarse que el entendimiento propio siempre sea limitado y hasta superficial (Piers, 1998). De forma concreta, y en términos muy generales, el inconsciente sería todo aquello que resulta inaccesible tanto para la persona como para el resto; al mismo tiempo, involucra una parte que resulta muy primitiva al ser humano y se ocupa de mantener oculta en lo profundo de la mente determinada información. Esto podría tratarse de material al cual nunca se pudiera acceder (Seelbach González, 2013).

Existen otros conceptos que resultan importantes a las teorías psicoanalíticas: la pulsión de vida y la pulsión de muerte (Eros y Thánatos, respectivamente) (Freud, 1913 1915), por una parte, y los mecanismos de defensa, por otro. En lo que refiere a la pulsión de vida se trata de una energía que incentiva al comportamiento a mantener la vida; al contrario de esto, la pulsión de muerte hace referencia a un tipo de energía destructiva, que conduce a la muerte. En cuanto a los mecanismos de defensa puede decirse que son aquellas estrategias que se ponen en marcha al momento de resolver el conflicto psíquico; éstos aparecen cuando el monto de energía del estímulo es muy fuerte para el superyó, por lo cual se torna peligros en la realidad del sujeto (Cloninger, 2003). A propósito de estos mecanismos de defensa 
cabe aclarar que son varios, pero que no serán explicados aquí ya que no resulta pertinente a los fines de este estudio, alcanza con saber acerca del concepto en sí mismo.

Si bien, la terminología es mucho más amplia en estas teorías, los conceptos expuestos antes sirven a los fines de dar cuenta la dinámica de la personalidad en estos enfoques. En ese sentido, puede decirse que las teorías psicodinámicas de la personalidad entienden a esta última como la interacción de los conceptos explicados anteriormente; si esta interacción es adecuada se puede esperar una personalidad que se adapte a los requerimientos de la realidad (la que depende del contexto). Contrariamente a esto, una interacción defectuosa supone la concepción de patología respecto de la personalidad. También, hay que destacar que, si bien los desarrollos más contemporáneos del psicoanálisis van más allá de las fijaciones sexuales tempranas, siguen manteniendo la misma dinámica.

\section{B. Teorías Humanístico-Existenciales (o Fenomenológicas)}

Estas teorías que consideran que los individuos deban ser entendidos como el resultado de dinámicas inconscientes, sino que la personalidad se construye a partir de perseguir y alcanzar metas que sean positivas, lo que implica que estén encaminadas a obtener logros, que al mismo tiempo tengan componentes benéficos
(Montaño et al., 2009; Morris \& Maisto, 2005).

En suma, Allport (1940) señala que los factores individuales son de gran importancia al momento de determinar la personalidad; explica que la motivación debe mantenerse continua a lo largo de la vida, y destaca una acción importante que es la de actuar con autonomía, ya entiende que en la medida que los motivos sean autónomos esto da cuenta del nivel de madurez. Al mismo tiempo, señala el lugar que adquiere el yo, concepto al que le confirió gran importancia a partir de entender su rol en las características más destacadas de la personalidad. Para que esta idea del yo no se confundiese con la concepción freudiana, desarrolla el concepto de proprium, que tiene que ver con la percepción que el sujeto tiene de sí mismo (Mischel, 1988).

También, las teorías que son parte de esta línea son innovadoras respecto a la incorporación de nuevos conceptos, tales como la autorrealización, la libertad, la esperanza, el amor, la espiritualidad, la felicidad, la satisfacción. Estos tipos de teorías se basan en las posibilidades que tiene el sujeto para su crecimiento personal (Seelbach González, 2013). Cloninger (2003) explica que aparecieron "para combatir las tendencias deterministas y fragmentarias del psicoanálisis y el conductismo" (p. 409). También enumera 
algunas características distintivas de este tipo de teorías:

I) Se centran en lo que consideran aspectos "superiores", que son a su vez más evolucionados y sanos en lo que refiere a las experiencias humanas y sus desarrollos; la tolerancia y la creatividad se encuentran entre los aspectos más distintivos;

II) Tienen en cuenta la experiencia subjetiva de cada individuo, incluyendo en esto la experiencia emocional (en este caso el enfoque es bien fenomenológico);

III) El énfasis está puesto preferentemente en el presente, más que en el futuro o el pasado;

IV) Resaltan que cada sujeto, como adulto, debe hacerse responsable sobre los resultados de sus actos, ya que la capacidad de autorreflexión es siempre la elección más sana y aporta al crecimiento personal;

V) Pretenden que los descubrimientos que vayan obteniendo mejoren la condición humana, a partir de aplicarlos en los ambientes en los cuales las personas se desarrollan. $\mathrm{Si}$ esto sucediese, como las condiciones serían las apropiadas, el desarrollo se daría en un sentido deseable.
Dentro de este tipo de teorías, una de las principales es la desarrollada por Carl Rogers (1954), quien concibe su modelo desde la noción de proceso, entendiendo al ser humano desde un lugar positivo; de esta manera, para Rogers, los seres humanos se construyen y se moldean según la propia percepción del self. Así, puede sintetizarse que la personalidad se desarrolla en función de las experiencias cotidianas, y teniendo en cuenta la capacidad de actualización que cada sujeto posee (Cloninger, 2003; Costa \& McCrae, 1995).

Otro de los autores relevantes en este movimiento es Abraham Maslow (1943), quien aporta la interpretación de la personalidad a partir de cuestiones motivacionales. Poniendo su foco en los valores, señaló que el desarrollo de la persona se da a través de diferentes niveles o jerarquías de necesidades: las fisiológicas, las de seguridad, las de pertenencia, las de estima y las de autorrealización; en cuanto a las primeras cuatro los sujetos se motivan a partir de deficiencias, mientras que en el caso de la restante la motivación viene del ser (Cloninger, 2003; Costa \& McCrae, 1995; Seelbach González, 2013).

A partir de esto, puede entenderse que para este tipo de teorías se impone una combinación de los aspectos antes mencionados, que dan cuenta de las características fenomenológicas en la construcción de la personalidad; es decir que ésta estaría conformada por la interacción de 
dichos factores, resaltando que estas características son distintivas y asumen un compromiso con la importancia del crecimiento personal. Por este motivo, se entiende que se centren en aspectos que consideran de orden superior, valorando siempre la experiencia subjetiva como componente central en la construcción de la personalidad, acentuando la importancia de centrarse en el presente.

\section{Teorías Cognitivas y del Aprendizaje Social}

Para este tipo de teorías la personalidad se constituye a partir de la interacción entre el ambiente y los procesos psicológicos. Las diferencias individuales se relacionan con la forma de comprender el medio, a los otros y a sí mismos; así, las cogniciones pasan a ser la base para entender esas diferencias, por lo tanto, la clave para el cambio de la personalidad se da a partir de la capacidad de generar un cambio cognoscitivo (Cloninger, 2003). Seelbach González (2013) sostiene que la personalidad de cada individuo estará determinada por los aprendizajes de su vida, los cuales se dan a partir del ambiente que proporciona información en interacción con los sistemas de creencias individuales y los procesos psicológicos. De esta manera queda de manifiesto la importancia, para este tipo de teorías, del contexto social.

Uno de los autores más representativos en este tipo de teorías es Bandura, quien sostiene que la personalidad es el resultado de la interacción entre cognición, ambiente y aprendizaje; también les da un papel central a las expectativas internas de los sujetos, ya que la influencia del ambiente en el comportamiento hace que esas expectativas se vayan modificando (Bandura, 1977). De esta forma, dependiendo de que se cumplan o no tales expectativas, quienes llegan a tener altos niveles de desempeño interno desarrollan autoeficacia, que se entiende como la capacidad de atravesar las diferentes situaciones, de manera adecuada, y así llegar a obtener los resultados esperados (Bandura y Locke, 2003).

Para especificar aún más lo expuesto por este autor, resulta importante señalar que su punto de vista trasciende la teoría más radical del aprendizaje (que sostenía que las respuestas debían ser reforzadas para que se fortalecieran), ya que incluye la idea de modelización, es decir, la importancia de los buenos modelos; para fundamentar esto va explicar que las personas aprenden a partir de observar, lo que supone transgredir lo que se dijo anteriormente: que el aprendizaje se da si hay reforzamiento. Por lo tanto, se puede decir que el aprendizaje es, también, resultado de estar expuestos a modelos, y esto se conoce como aprendizaje vicario (o lo que es igual: aprendizaje observacional o aprendizaje imitativo) (Bandura, 1965; Cloninger, 2003).

Por su parte, Beck et al. (1995) comenta que el carácter tiene una tendencia 
a formarse de manera continua y a partir de los procesos más básicos de socialización; esto es así porque se define según las dimensiones que se aprenden y se vinculan a los factores psicosociales. Entre los modelos que se centran en las dimensiones del carácter pueden destacarse aquellos que se focalizan en la idea de 'esquema', de los cuales se entiende que son estructurados y que se encuentran entrelazados, y que son responsables de procesar los estímulos recibidos, así como la respuesta conductual a la que se asocia.

Otro de los autores que está incluido en este tipo de teorías es Mischel, quien se interesó en estudiar las variables personales del aprendizaje social cognitivo, más precisamente aquellas que dieron cuenta de otras formas de percibir las diferencias conductuales; complementando a lo explicado en los párrafos anteriores, los estudios realizados en esta línea permitieron dar cuenta de cómo las características de un individuo también influyen en las distintas situaciones así como en el ambiente, y se pudo corroborar que cada sujeto tiene ciertos patrones de conducta que son complejos así como diferentes para cada interacción cotidiana (Mischel, 1973). El autor explica que por estos motivos resulta importante identificar cuáles son las aptitudes individuales que permiten al sujeto construir diferentes conductas bajo determinadas condiciones; de igual forma, es necesario considerar tanto la codificación como la clasificación que realiza de la situación.
En función de lo planteado hasta aquí, puede decirse que las teorías que conforman este tipo de enfoque sostienen que no sería posible llegar a un modelo de la personalidad que sea adecuado si sólo se tienen en cuenta las conductas manifiestas sin observar lo que se piensa. En ese sentido, puede decirse que las teorías de la personalidad de esta línea estudian los efectos que tienen en la conducta los diferentes procesos mentales. Los teóricos de este enfoque hacen hincapié en las variables cognitivas justamente por la importancia que le dan al hecho de que la capacidad humana de pensar tiene al momento de estructurar la personalidad.

\section{Teorías Biológicas y del Rasgo}

Estas teorías hacen referencia a las características más particulares de cada persona, tales como la forma de adaptarse, el temperamento y los valores que hacen que el sujeto se desempeñe en torno a una característica en particular (Cloninger, 2003; Engler, 1996). En términos generales, las teorías incluidas en esta perspectiva difieren ampliamente entre sí; no obstante, Según Cloninger (2003), un par de coincidencias en algunas suposiciones que son básicas:

a) En general, este tipo de teorías se interesan enfáticamente en las diferencias individuales que existen en aquellas características que son relativamente estables a lo largo del tiempo y las situaciones; 
b) Tienen un especial interés en la medición de los rasgos a partir del uso de tests.

$\mathrm{Si}$ bien, quienes desarrollan estas teorías aún no han logrado un acuerdo sobre cuáles serían las dimensiones más importantes de la personalidad, se siguen debatiendo los puntos teóricos referentes a los rasgos. Esto ha dado lugar a la aparición de un nuevo paradigma sobre el cual se argumenta que el desarrollo sobre los rasgos es lo que permitiría establecer la cohesión que estas teorías buscan, y al mismo tiempo permitiría enmarcar la teoría de la personalidad en la tradición de las ciencias naturales. Esto último, resulta de especial interés ya que acerca esta ciencia al resto de las ciencias, debido a que para estas últimas la taxonomía aparece antes que el análisis causal (Cloninger, 2003; Eysenck, 1991).

Resumiendo, esta idea, y retomando lo antes dicho, "los rasgos representan disposiciones estables del comportamiento, tendencias a actuar de manera relativamente consistente, y son permanentes a lo largo de la vida" (Sánchez \& Ledesma, 2007, p. 5).

\section{Los cinco grandes de la personalidad}

En la actualidad el Modelo de los Cinco Grandes, o Modelo de los Cinco Factores, es uno de los que más aceptación ha alcanzado en lo que refiere a teorías de la personalidad, y propone la descripción de esta misma a partir de cinco dimensiones: extraversión (E), neuroticismo $(\mathrm{N})$, conciencia (C), agradabilidad (A) y apertura a la experiencia $(\mathrm{O})$, considerando que se trata de rasgos presentes en todos los individuos, a partir de lo que se infiere su carácter universal (Cassaretto, 2010). En función de esto, este modelo entiende a la personalidad como los estilos individuales, motivacionales, emocionales, experienciales e interpersonales que permite diferenciar las características individuales (Cassaretto, 2010; Costa \& McCrae, 1992). Sin embargo, antes de la conformación de esta teoría, la perspectiva de los rasgos no gozaba de tanto crédito, debido a que se observaba cierto grado de desorganización (Funder, 2001).

Se trata de una teoría que se basa en el enfoque léxico de la personalidad, a partir del cual se consideran la existencia de cinco factores amplios, ya que estos a su vez engloban -cada uno- más dimensiones, sobre los cuales puede ser abarcada la mayor parte de las características de la personalidad, de manera independiente al lenguaje y la cultura, y que resultan estables al paso del tiempo; en ese sentido, es importante aclarar que la estructura de este modelo no supone que las diferencias en la personalidad se reduzcan a ella (John \& Srivastava, 1999; McCrae \& Costa, 1990). Al mismo tiempo, al ser consideradas por muchos autores como las principales dimensiones de la personalidad se constituyen en un cimiento explicativo de esta (John, 1990). 
Por otro lado, un aspecto a señalar en cuanto esta teoría es que la noción de rasgo adoptada no es la misma acuñada por Allport, ya que, si bien fue impulsor del concepto, sostenía que aquello que debiera interesar a la Psicología de la Personalidad son las características de carácter individual, y no buscar rasgos en común entre individuos para luego compararlos; de hecho, rechazaba los desarrollos factoriales (Romero, 2002; Sánchez \& Ledesma, 2009). Por el contrario, el Modelo de los Cinco Grandes -como se dijo- encuentra su fundamento a partir de tener en cuenta estos cinco factores, considerados amplios, que pueden explicar de manera abarcativa los rasgos de la personalidad; estos factores aparecen luego de realizar variados análisis sobre datos que ya existían. Además, esta solución de cinco factores parece un punto medio adecuado entre lo desarrollado por Eysenck (tres factores), que parecía poco, y lo hecho por Cattell (16 factores), que suponía demasiado. Por este motivo parece justa la defensa de los autores que adhieren a esta forma de explicar la personalidad, ya que en este modelo se puede contemplar la base genética de los rasgos, así como su consistencia transituacional, la estabilidad temporal y la estructura universal (McCrae \& Costa, 1990; Romero, 2005; Sánchez y Ledesma, 2009).

A continuación, se presenta la descripción de los factores que componen el Modelo de los Cinco Grandes, incluyendo lo que Costa y McCrae llaman factores de segundo orden o facetas. Para esto, se recupera el resumen propuesto por Sánchez y Ledesma (2009), y se describen los principales factores, $\mathrm{y}$ en sus respectivas las facetas:

- Amabilidad (o cordialidad, y su opuesto es Antagonismo): hace referencia a la capacidad de poder establecer vínculos sociales, así como a la disposición de preocuparse por los demás; en cuanto su opuesto se relaciona a la psicopatía. Esta es una dimensión que no siempre aparece en todos los modelos; un motivo para explicar esto es que tiene un carácter valorativo.

- Confianza: es la capacidad para atribuir a los otros buenas intenciones en sus actos.

- Franqueza: incluye a las personas sinceras, e incluso algo ingenuas.

- Altruismo: son aquellos sujetos generosos, que muestran una preocupación genuina por los demás.

- Actitud conciliadora: suelen perdonar ofensas, son cooperativos y evitan los conflictos.

- Modestia: tiene que con la conducta de pasar desapercibido.

- Sensibilidad social: se asocia a los sentimientos de solidaridad y de piedad.

- Responsabilidad (o Escrupulosidad, y su opuesto es Irresponsabilidad o 
Negligencia): en este caso se refiere a la capacidad que tiene el individuo de actuar conforme a metas claras o propósitos establecidos, lo que le permite organizarse y poder llevar a cabo diferentes ideas o proyectos. El opuesto incluye a quienes no tienen un rumbo determinado, también resultan personas indolentes. Al igual que la dimensión anterior, ésta también puede estar ausente en algunos modelos, e incluye el control de los impulsos en sentido de evidenciar disposición a ser obediente y escrupuloso.

- Competencia: sentirse apto y resolutivo en diferentes aspectos de la vida.

- Orden: estar bien organizado, es decir la tendencia a ser ordenado.

- Sentido del deber: tiene que ver con la adherencia del sujeto a sus principios éticos y a cumplir con obligaciones.

- Necesidad de logro: es parte de aquellas personas que tienen niveles altos de aspiraciones, y que trabajan duro para conseguir eso que quieren.

- Autodisciplina: es la capacidad para empezar y también terminar tareas, aunque le resulten aburridas, o si aparece algún tipo de distracción.

- Reflexión: tiene que ver con la habilidad de pensar con cuidado antes de actuar.
- Extraversión (y su opuesto es la Introversión): en algunos modelos también se la llama Sugerencia, y evalúa la sociabilidad en el sentido de comunicarse fácilmente con los otros; también tiene que ver con la asertividad y la capacidad para iniciar y mantener conversaciones con los demás; en el extremo opuesto se encuentran quienes muestran retraimiento o una tendencia a aislarse.

- Cordialidad: son aquellas personas quienes son capaces de establecer relaciones amables con los demás.

- Gregarismo: cuando prefieren estar en compañía de los otros.

- Asertividad: son aquellas personas que tienen facilidad para decir lo que piensan, incluso cuando no están de acuerdo; también contempla la tendencia de ser seguro de sí mismo.

- Actividad: son aquellos sujetos que siempre están buscando para hacer; la característica distintiva son las conductas motoras vigorosas.

- Búsqueda de emociones: tiene que ver con la capacidad de disfrutar cosas nuevas, así como también a la capacidad de poder acercarse a lo que serían fuentes de estimulación.

- Emociones positivas: son quienes tienden, de manera frecuente, a tener experiencias con emociones 
tales como la felicidad, optimismo, alegría, entusiasmo.

- Neuroticismo (y su opuesto es la Estabilidad Emocional): se refiere a aquellos aspectos que se asocian al bienestar o malestar psicológico, tanto como todo aquello que se relaciona con emociones negativas, tales como tristeza, enojo, sentimientos de culpa y miedo; en ese sentido, también evalúa la inestabilidad emocional.

- Ansiedad: tiene que ver con aquellos sujetos que tienden a ser tensos o estar nerviosos, muy preocupados o temerosos.

- Hostilidad: son aquellos individuos que experimentan enfado, irritación.

- Depresión: se asocia a los sentimientos de desesperanza, tristeza, soledad, culpa.

- Timidez (o ansiedad social): se trata de quienes sienten incomodidad en situaciones sociales, son sensibles al ridículo o sienten vergüenza.

- Impulsividad: indica dificultades en torno al control de los impulsos y las necesidades, así como poca tolerancia a la frustración.

- Vulnerabilidad (al estrés): se asocia a la dificultad para tener control en situaciones estresantes, y también a ser dependiente de otros en aquellas situaciones que se perciben como de emergencia.
- Apertura a la experiencia (su opuesto es Convencional o Cerrado a la experiencia: se asocia con aquellos individuos que desarrollan la capacidad de ser introspectivos, son curiosos intelectualmente, tienen imaginación activa y sensibilidad estética; su opuesto evalúa la tendencia de apegarse a lo tradicional, el dogmatismo y lo convencional.

- Fantasía: se asocia a la capacidad de imaginar y de crear.

- Estética: se refiere a la capacidad apreciar la belleza y las cuestiones artísticas.

- Sentimientos: evalúa la capacidad para percibir los sentimientos $\mathrm{y}$ emociones propios, a partir de entenderlos como importantes.

- Acciones: indica intereses orientados a distintas actividades, rechaza lo convencional y la rutina.

- Ideas: se asocia al interés por las ideas poco convencionales, así como a los argumentos intelectuales.

- Valores: tiene que ver con la capacidad de examinar y evaluar los valores sociales, políticos o religiosos.

Una vez explicado esto, es conveniente resaltar que más allá de las diferencias en los análisis factoriales que se realicen en los distintos contextos, en términos generales puede obtenerse como 
resultado una factorización que da cuenta de la presencia de los cinco grandes, lo que apoya su solidez a la hora de evaluar y describir la personalidad.

\section{Conclusiones y consideraciones finales}

A partir de lo desarrollado supra puede observarse que el estudio de la personalidad resulta muy extenso; para quienes se dedican al estudio de la misma reconocen en esto una trayectoria que data de mucho tiempo, de hecho, está entre las primeras variables psicológicas que han despertado el interés de aquellos que investigan en Psicología. Asimismo, cada autor ha buscado dar una perspectiva integrada, pero el límite ha quedado en cada forma de interpretar este constructo, ya que aún se sigue observando una gran cantidad de trabajos, que responden a formas específicas de entender la personalidad, pero todavía no se logra un consenso acerca de cómo definirla de forma unívoca. Como puede verse reflejado a lo largo de este trabajo, esto podría deberse también a que en estas diferentes posturas -desarrolladas a través de los años- las perspectivas elaboradas responden tanto a diferentes criterios, como definiciones y categorías, las cuales no llegan a quedar delimitadas dentro de una idea unificadora. No obstante, si bien existe una idea de integrar, como sucede con la teoría de los cinco grandes, esta no termina de ser inclusiva de todos los aspectos a considerar. Queda claro que describe lo necesario acerca de las características de la personalidad, pero hay que tener en cuenta que lo hace de manera muy general.

En suma, cabe destacar que es esta característica de heterogeneidad lo que también hace que la personalidad siga siendo una variable de interés para su estudio, no solo el hecho de ser un constructo sumamente necesario para la descripción de las conductas, ya que esto último es una condición estable, es decir: siempre va a ser necesario describir la personalidad, y lo que sucede es que siempre será necesario generar indicadores que se ajusten a una descripción lo más ajustada posible del comportamiento del sujeto y su constitución estructural.

Para finalizar, hay que dejar claro que este trabajo no contempla todos los desarrollos acerca de esta variable, por lo tanto, no logra ser exhaustivo. Sin embargo, trata de lograr una línea que se encamine hacia una idea unificada de entender este constructo, buscando un punto de cohesión entre diferentes desarrollos. Se espera poder profundizar en estos aspectos, de manera de poder lograr una idea totalizadora acerca de cómo entender esta variable y sus diferentes componentes.. 


\section{Referencias}

Allport, G. (1970 a). La personalidad. Barcelona: Herder.

Allport, G. (1970 b). Psicología de la personalidad. Buenos Aires: Paidós.

Allport, G. W. (1937). Personalidad: una interpretación psicológica. Oxford, Inglaterra: Holt.

Allport, G. W. (1966). Rasgos revisados. Psicólogo Estadounidense, 21(1), 110. doi: $10.1037 / \mathrm{h} 0023295$

Bandura, A. \& Locke, E. (2003). Negative Self-efficacy and goal effect revisited. Journal of Applied Psycholgy, $8,87-99$

Bandura, A. (1965). Influence of models' reinforcement contingencies on the acquisition of imitative responses. Journal of Personality and Social Psychology, 1(6), 589-595.

Bandura, A. (1977). Social learning theory. Englewood Cliff: Prentice Hall.

Beck, A., Freeman, A., Pretzer, J., Davis, D., Fleming, B. \& Ottaviani, R. (1995). Terapia cognitiva de los trastornos de personalidad. Barcelona: Paidós.

Bermúdez, J. (1999). Personality and health protective behaviour. European Journal of Personality, 13, 83-103.
Brenlla, M. E. (1996). Evaluación objetiva de la personalidad: obtención de normas regionales para el Inventario Multifacético de la Personalidad de Minnesota - 2. Revista Argentina de Clínica Neuropsiquiátrica, 3.

Cassaretto, M. (2011). Relaciones entre la personalidad y el afrontamiento en estudiantes preuniversitarios. Vanguardia Psicológica, 1(2), 202225 .

Castro Solano, A. \& Casullo, M. M. (2001). Rasgos de personalidad, bienestar psicológico y rendimiento académico en adolescentes argentinos. Interdisciplinaria, 18(1), 65-85.

Castro Solano, A. (2015). La aproximación teórico-racional: el modelo de $\mathrm{T}$. Millon en M. Fernández Liporace y A. Castro Solano (Comp.)., Evaluación de la personalidad normal y sus trastornos (pp.15-47). Buenos Aires: Lugar.

Cattell, R. B. (1979). Personality and learning theory, Vol. 1: The structure of personality in its environment. Nueva York: Springer.

Cerda, E. (1985). Una psicología de hoy. Barcelona: Herder. 
Chico Librán, E. (2015). Manual de Psicología de la Personalidad. Alicante: Editorial Club Universitario.

Cloninger, S. C. (2003). Teorías de la personalidad. México D.F.: Pearson Educación.

Costa, P. \& McCrae, R. (1995). Primary traits of Eysenck's P-E-N system: Three-and Five factor solutions. Journal of Personality and Social Psychology, 69, 308-317.

Costa, P. T., \& McCrae, R. R. (1992). Four ways five factors are basic. Personality and Individual Differences, 13, 653-665.

Costa, P. T., Jr., \& McCrae, R. R. (1985). The NEO Personality Inventory manual. Odessa, FL: Psychological Assessment Resources.

Engel, B. (1996). Teorías de la personalidad. México D.F.: Mc Graw Hill.

Errasti Pérez, J. (1998). Usos y abusos de la Psicología de Eysenk. Psicothema, 10(3), 517-533.

Eysenck, H. (1947). Dimensions of Personality. Londres: Routledge \& Ke-gan Paul.

Eysenck, H. J. (1991). Dimensions of personality: 16, 5, 3? Personality and Individual Differences, 12, 773-790.
Freud, S. (1913-1915). Sigmund Freud. Obras completas (Volumen XIV). Buenos Aires: Amorrortu Editores (1976).

Freud, S. (1920-1922). Sigmund Freud. Obras completas (Volumen XVIII). Buenos Aires: Amorrortu Editores (1976).

Funder, D. C. (2001). Personality. Annual Review of Psychology, 52, 197-221.

John, O. P. (1990). The "Big Five" factor taxonomy: Dimensions of personality in the natural language and in questionnaires. En L. A. Pervin (Ed.), Handbook of personality: Theory and research (pp. 66-100). New York: Guilford.

John, O. P., \& Srivastava, S. (1999). The Big Five Trait taxonomy: History, measurement, and theoretical perspectives. En L. A. Pervin \& O. P. John (Eds.), Handbook of personality: Theory and research (pp. 102-138). New York, NY, US: Guilford Press.

Jung, C. G. (1954). Marriage as a psychological relationship. In C. G. Jung, The development of personality (pp. 187-201) (W. McGuire, Ed.; R. F. C. Hull, Trans.). Princeton, NJ: Princeton University Press. (Original work published 1931).

Klein, M. (1946). Notes on some schizoid mechanisms. In M. Klein, P. 
Heimann, S. Issacs, \& J. Riviere (Eds.)., Developments in psychoanalysis. London: Hogarth Press.

Leal, I., Vidales, F. \& Vidales, I. (1997). Psicología General. México D.F.: Limusa.

Lluís Font, J. M. (2002). Personalidad: esbozo de una teoría integradora. Psicothema, 14(4), 693-701.

Maslow, A. H. (1943). A theory of human motivation. Psychological Review, 50, 370-396. Reprinted in R. J. Lowry (Ed.), Dominance, self-esteem, selfactualization: Germinal papers of A. H. Maslow (1973, pp. 153-173). Monterey, CA: Brooks/Cole.

McCrae, R.R., y Costa, P.T. (1987). Validation of the Five-Factor model of personality across Instruments and observers. Journal of Personality and Social Psychology, 52, 81-90.

Mikulic, I. M. (s.f.). Inventario Multifacético de Personalidad de Minnesota: guía para la administración e interpretación del MMPI-2. Material de cátedra: Teoría y Técnica de Exploración y Diagnóstico. Facultad de Psicología. Universidad de Buenos Aires.

Millon, T. \& Everly, G. (1994). La personalidad $y$ sus trastornos. Barcelona: Martínez Roca.
Mischel, W. (1973). Toward a cognitive social learning reconceptualization of Personality. Psycological Review, 80, 252-283.

Mischel, W. (1988). Teorías de la personalidad. México D.F.: Mc Graw Hill

Montaño Sinisterra, M., Palacios Cruz, J. \& Gantiva Díaz, C. (2009). Teorías de la personalidad. Un análisis histórico del concepto y su medición. Psychologia. Avances de la Disciplina, 3(2), 81107.

Morris, G. \& Maisto, A. (2005). Psicología. México D.F.: Prentice Hall.

Orengo Caus, T., González Abolafio, M., Benito Delegido, A., Ballester Sánchez, F., Cervera Martínez, C. \& Haro Cortez, Y. G. (2007). El modelo psicobiológico de Cloninger en dependientes de Opiáceos. Trastornos Adictivos, 9(2), 108-115.

Pervin, L. \& John, O. (1998). Personalidad: teoría e investigación. México D.F.: Manual Moderno.

Piers, C. (1998). Contemporary trauma theory and its relation to character. Psychoanalytic Psychology, 15, 1433.

Real Academia Española. (2017). Definición de personalidad. Recuperado de: 
http://dle.rae.es/srv/search?m=30\&w =personalidad

Rogers, C. R. (1954). Towards a theory of creativity. ETC: A Review of General Semantics, 11, 249-260.

Rogers, C. R. (1964). Toward a modern approach to values: The valuing process in the mature person. Journal of Abnormal and Social Psychology, $68,160-167$

Romero, E. (2002). Investigación en Psicología de la Personalidad. Líneas de evolución y situación actual. Boletín de Psicología, 74, 39-77.

Sánchez, R. O. \& Ledesma, R. (2007). Los cinco grandes factores: cómo entender la personalidad y cómo evaluarla. En A. Mojeau (Ed.)., Conocimiento para la transformación. Serie Investigación y Desarrollo (pp. 131160). Argentina: Ediciones Universidad Atlántida.

Schmitt, D. P., Allik, J., McCrae, R. R. \& Bennet Martínez, V. (2007). The geographic distribution of Big Five
Personality traits. Patterns and profiles of human self description across 56 nations. Journal of Cross Cultural Psychology, 38(2), 173-212. doi: 10.1177/0022022106297299

Seelbach González, G. A. (2012). Teorías de la Personalidad. México D.F.: Red Tercer Milenio.

Sullivan, H. S. (1953). The interpersonal theory of psychiatry. New York: Norton.

Wilt, J. \& Revelle, W. (2015). Afecto, comportamiento, cognición y deseo en los cinco grandes: un análisis del contenido y la estructura del artículo. European Journal of Personality, 29(4), 478-497. doi: 10.1002/per.2002.Docencia Creativa, 2, 54-62. Recuperado de http://digibug.ugr.es/bitstream/handle /10481/27621/ReiDoCrea-Vo1.3Art.7\%ADRevelli-Gutierrez-CastilloCenteno-Vinuesa-BelcaidAndrade.pdf:jsessionid $=5556 \mathrm{E} 56 \mathrm{C} 90$ 3351B415895D12E0F42484?sequen $\underline{c e=1}$. 